Proceeding Series of the Brazilian Society of Computational and Applied Mathematics

\title{
Hybridization of IMC and PID Control Structures from Filtered Positional Generalized Predictive Controller
}

\author{
Rejane de Barros Araújo ${ }^{1}$ \\ Daniel Cavalcanti Jeronymo² \\ Antonio Augusto Rodrigues Coelho ${ }^{3}$ \\ Federal University of Santa Catarina \\ Department of Automation and Systems 88040-900 \\ Florianopolis, SC, Brazil
}

\begin{abstract}
The purpose of this paper is to derive an alternative tuning design from Generalized Predictive Controller (GPC) that is based on both positional plant model and cost function, involving the selection of an integral polynomial weighting filter. The obtained Filter Positional GPC can be transformed into RST structure and next to Filtered Internal Model and PID controllers. Numerical and experimental essays show the effectiveness of the control methodologies.
\end{abstract}

Keywords. predictive control, PID controller, tuning, target tracking, stability analysis.

\section{Introduction}

The Generalized Predictive Controller (GPC) has been successfully implemented in several applications, showing good robustness, performance and dynamic stability $[2,4]$.

Hybridization of GPC to the Internal Model Control (IMC) structure has stimulated academia studies. However, few discrete implementations have been explored and aimed to extract the characteristics and advantages of the GPC to IMC in order to deal with a variety of complex processes and disturbance rejection. The work proposed in [5] has shown the GPC design with different types of constraints relating to the IMC control law, inserting anti-windup technique in numerical simulation scenarios.

On the other hand, Proportional-Integral-Derivative (PID) controllers are widely used in the industry as standalone equipment or in CLP. The pioneer work using PID in the structure I+PD controller hybridized with GPC was proposed in [6], using a design method of a self-tuning PID controller. Next, [8] has extended the work of [6], inserting a filter in the reference of the GPC law, where the PID controller has a filter to tune the integral time of the controller.

\footnotetext{
${ }^{1}$ rejane.barros@posgrad.ufsc.br

2d.cavalcanti@posgrad.ufsc.br

3 antonio.arc@ufsc.br
} 
The purpose of this paper is to derive a design alternative for the GPC synthesis, from a positional plant model, involving the selection of an integral polynomial weighting factor for reference and plant output signals. This control scheme is called filtered positional GPC (FP-GPC). The FP-GPC is transformed into two degree of freedom polynomial RST structure and next to Filtered Internal Model (F-IMC) and PID controllers. The idea is to inherit the FP-GPC properties (stability, uncertainty, setpoint tracking) to the PID and F-IMC control structures, investigating applications in different types of plants.

This paper is organized as follows: Section 2 presents briefly the FP-GPC design. Sections 3 and 4 describe how the FP-GPC controller, in the RST form, is convert to F-IMC and PID control schemes, respectively. Numerical and practical simulations are also included. Finally, conclusions are given in Section 5.

\section{Filtered Positional GPC Design}

Consider the deterministic CAR model (Controlled Auto-Regressive) of the controlled plant characterized by the following positional discrete transfer function:

$$
A\left(q^{-1}\right) y(t)=q^{-d} B\left(q^{-1}\right) u(t-1)
$$

where $y(t)$ is the process output, $u(t)$ is the control signal, $d$ is the dead-time and the roots of the polynomials $A\left(q^{-1}\right)$ and $B\left(q^{-1}\right)$ characterize the open-loop poles and zeros, respectively. The GPC control law is obtained by minimizing the cost function given by

$$
J=\sum_{j=1}^{N_{y}}\left\{\phi_{y}(t+j)-\phi_{w}(t+j)\right\}^{2}+\lambda \sum_{j=1}^{N_{u}} u^{2}(t+j-d-1)
$$

with $\phi_{y}(t+j)$ and $\phi_{w}(t+j)$ auxiliary output and reference variables and are defined as

$$
\phi_{y}(t)=P\left(q^{-1}\right) y(t)=\frac{K_{\alpha} \alpha\left(q^{-1}\right)}{\Delta} y(t) \quad, \quad \phi_{w}(t)=P\left(q^{-1}\right) w(t)=\frac{K_{\alpha} \alpha\left(q^{-1}\right)}{\Delta} w(t)
$$

where $w(t)$ is the setpoint, $\Delta=\left(1-q^{-1}\right), \lambda$ is the control weighting, $N_{y}$ is the output prediction horizon and $N_{u}$ is the control horizon. Polynomials $P\left(q^{-1}\right)$ and $\alpha\left(q^{-1}\right)$ are correlated with closed-loop system dynamic and filtering aspects, respectively, and $K_{\alpha}$ represents the filter gain.

The term $\phi_{y}(t+j)$ is replaced by the estimated value, the equation (1) is multiplied by $P\left(q^{-1}\right)$ and can be rewritten as

$$
\Delta A\left(q^{-1}\right) \phi_{y}(t+j)=K_{\alpha} \alpha\left(q^{-1}\right) B\left(q^{-1}\right) u(t+j-d-1)
$$

The minimization of the cost function generates the FP-GPC for the unconstrained case, and the control vector is calculated by

$$
U=\left(G^{T} G+\lambda I\right)^{-1} G^{T}\left[\Phi_{w}-\Phi_{f}\right]
$$


which is a similar formalism of the incremental GPC design of [2] for the incremental fixed structure. To analyze the influence of the filter $P\left(q^{-1}\right)$ for setpoint changes and disturbance attenuation control purposes, the equation (4) can be rewritten to the polynomial RST canonical form such as

$$
\left[1+q^{-1} \sum_{j=1}^{N_{y}} k_{j} \bar{G}_{j}\right] u(t)=\sum_{j=1}^{N_{y}} k_{j} q^{j} \phi_{w}(t)-\sum_{j=1}^{N_{y}} k_{j} F_{j} \phi_{y}(t)
$$

where $K$ is the vector obtained from the first row of the matrix $\left(G^{T} G+\lambda I\right)^{-1} G^{T}$. Next, using the definition of $P\left(q^{-1}\right)$, then the RST canonical structure of the FP-GPC is given by

$$
R\left(q^{-1}\right) \Delta u(t)=K_{\alpha} \alpha\left(q^{-1}\right) T\left(q^{-1}\right) w(t)-K_{\alpha} \alpha\left(q^{-1}\right) S\left(q^{-1}\right) y(t)
$$

The block diagram of the RST structure for the FP-GPC design is shown in Figure (1). Reference, output and control filters are observed in a two degree of freedom control scheme, where $R\left(q^{-1}\right)$ and $S\left(q^{-1}\right)$ are designed to obtain the desired regulatory performance (disturbance rejection) and $T\left(q^{-1}\right)$ is designed to guarantee reference tracking (both polynomials depend on how the tuning parameters of the FP-GPC are selected).

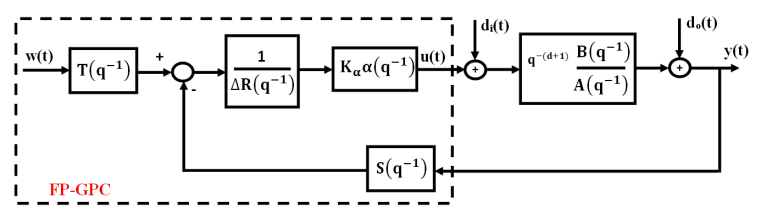

Figure 1: Disturbance on the input and output under FP-GPC system diagram.

Although not shown, it is important to say that for all simulation results of this paper, the filter parameters $K_{\alpha}$ and $\alpha\left(q^{-1}\right)$ of the FP-GPC are calibrated through a multiobjective optimization algorithm based on the sensitivity function $\left(M_{s} \triangleq \max _{0 \leq \omega<\infty} \mid A\left(e^{-j \omega}\right)\right.$ $\left.\Delta R\left(e^{-j \omega}\right) /\left(A\left(e^{-j \omega}\right) \Delta R\left(e^{-j \omega}\right)+K_{\alpha} \alpha\left(e^{-j \omega}\right) S\left(e^{-j \omega}\right) B\left(e^{-j \omega}\right)\right) \mid\right)$ and Integrated Absolute Error criterion $\left(I A E=\sum_{k=1}^{\infty}\left|e\left(k T_{s}\right)\right|\right)$, searching $1.2<M_{s}<2.0$ and a minimum value for IAE [7].

It is noteworthy that the FP-GPC design is an alternative synthesis to the standard formalism of the GPC of [2] and more details of the FP-GPC design can be found in [1].

\section{Filtered IMC Design from the FP-GPC}

This section presents the hybridization of the FP-GPC controller to the filtered polynomial structure of the RST canonical form and next to the Filter IMC (F-IMC) controller.

The hybridization design proposed in this paper is to extend the possibilities of the IMC controller tuning, deriving an new formalism to the conventional IMC structure with the FP-GPC to avoid instability problems and to provide satisfactory robustness and performance of the controlled system. In addition, dealing with disturbance and modelplant mismatch, even for unstable and non-minimum phase processes. 
Figure (1) shows the structure of the FP-GPC controller with a filter $K_{\alpha} \alpha\left(q^{-1}\right)$ in the direct loop where $S\left(q^{-1}\right) \neq T\left(q^{-1}\right)$ and the controller is $C_{R S T}\left(q^{-1}\right)=K_{\alpha} \alpha\left(q^{-1}\right) / \Delta R\left(q^{-1}\right)$.

Another way to represent the FP-GPC is to use the control structure of Figure (1), converting to the control structure of F-IMC, and the block diagram is shown in Figure (2).

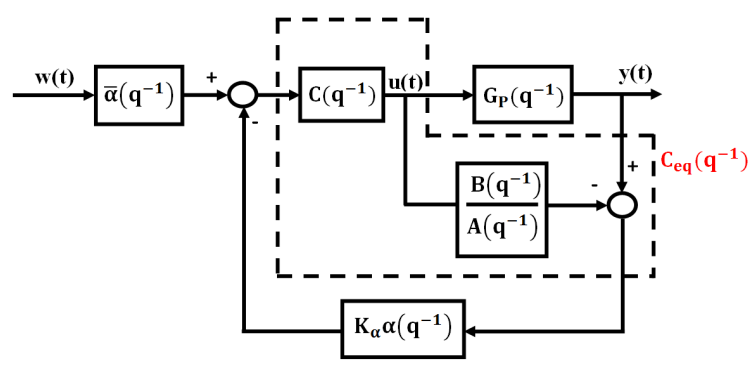

Figure 2: Two degree of freedom of the F-IMC.

The F-IMC system of Figure (2) and FP-GPC of Figure (1) will be equivalent if the controller $C\left(q^{-1}\right)$ in the F-IMC block diagram is chosen according to

$$
C\left(q^{-1}\right)=\frac{S\left(q^{-1}\right) A\left(q^{-1}\right)}{A\left(q^{-1}\right) \Delta R\left(q^{-1}\right)+K_{\alpha} \alpha\left(q^{-1}\right) S\left(q^{-1}\right) B\left(q^{-1}\right)} \quad, \quad \bar{\alpha}\left(q^{-1}\right)=\frac{K_{\alpha} \alpha\left(q^{-1}\right) T\left(q^{-1}\right)}{S\left(q^{-1}\right)}
$$

The FP-GPC turned into F-IMC structure is different from [5] control synthesis, because the design formalism of the proposed F-IMC uses a plant model and cost function in positional forms, there is an integral polynomial error weighting and ensures good closedloop stability. The idea is to inherit the FP-GPC properties to the IMC scheme, opening possibilities of applications in hard conditions for different kinds of plant dynamics.

\subsection{Simulation Results to a Third-Order Process}

This essay is based on a numerical simulation of an unstable and non-minimum phase system. Plant and model transfer functions are given by

$$
G_{p}\left(q^{-1}\right)=\frac{-0.006 q^{-1}+0.013 q^{-2}+0.008 q^{-3}}{1-2.25 q^{-1}+1.55 q^{-2}-0.32 q^{-3}} \quad, \quad G_{m}\left(q^{-1}\right)=\frac{0.007 q^{-1}+0.015 q^{-2}}{1-1.89 q^{-1}+0.87 q^{-2}}
$$

Figure (3) shows the time response of the FP-GPC and F-IMC control algorithms, for step setpoint changes and load disturbances (10\% of the setpoint magnitude value at time instants $110 \mathrm{~s}$ and $180 \mathrm{~s}$ ) applied to the input and output of the plant. The controllers have equivalent performance and good closed-loop dynamic, that is depending on the choice of the filter tuning parameters. The controller is tuned with $N_{y}=20, N_{u}=2, \lambda=8$, $K_{\alpha}=0.2329, \alpha\left(q^{-1}\right)=\left(1-0.0776 q^{-1}\right)$ and sampling period of $0.5 \mathrm{~s}$.

As noted in Figure (3), the FP-GPC and F-IMC controllers are equivalent and can remove the input and output disturbances. In addition, the numerical simulation shows that the hybridization method, proposed in this paper, can be applied in a variety of plants in hard conditions. 

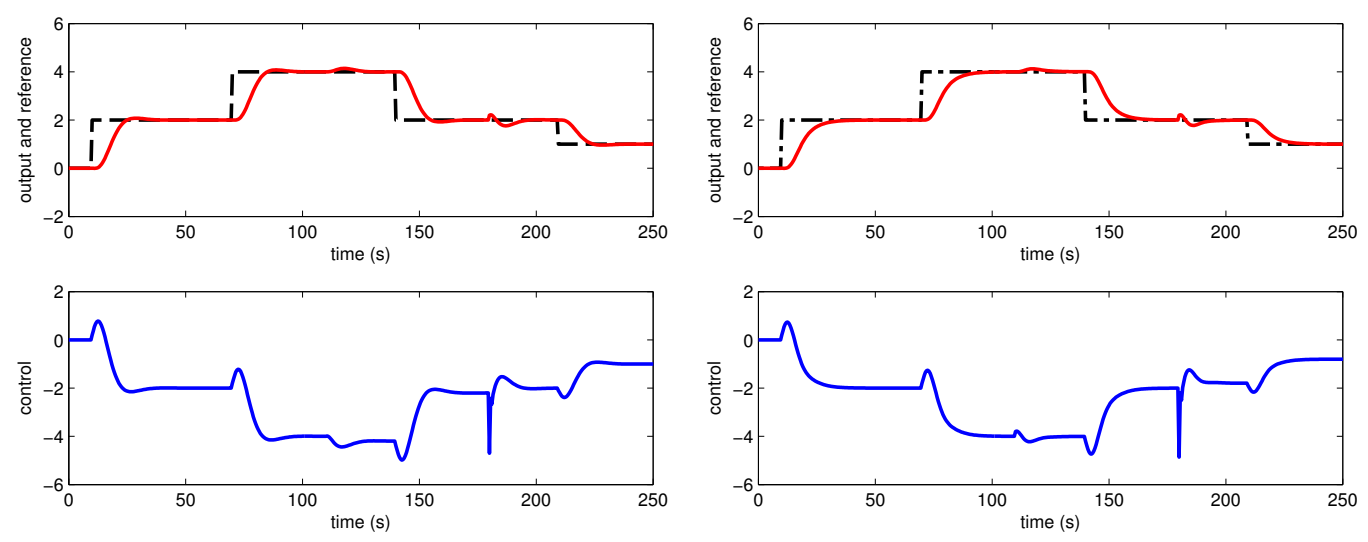

(a) FP-GPC

(b) F-IMC

Figure 3: System response to FP-GPC and F-IMC controllers.

\section{PID Design from the FP-GPC}

This section presents the hybridization of the FP-GPC controller to the PID controller. The FP-GPC design is used to generate an optimal calibration for the PID gains. The PID controller implemented in this paper is as follows: i) the integral term uses $e(t)=$ $w(t)-y(t)$; ii) the proportional and derivative terms use $e(t)=-y(t)$. Therefore, the PID controller in a digital form, is written as

$$
\Delta u(t)=K_{c} \frac{T_{s}}{T_{i}} \omega(t)-K_{c}\left(1+\frac{T_{s}}{T_{i}}+\frac{T_{d}}{T_{s}}\right) y(t)+K_{c}\left(1+2 \frac{T_{d}}{T_{s}}\right) y(t-1)-K_{c} \frac{T_{d}}{T_{s}} y(t-2)
$$

that represents the control structure called I+PD [3]. The constants $K_{c}, T_{i}, T_{d}$ are the proportional gain, the integral time and the derivative time, respectively, and $T_{s}$ is the sampling period.

Next, the FP-GPC controller design is combined with the PID control law of equation (9) to ensure not only the dynamic properties of the closed-loop FP-GPC but also to provide a robust stability with good set of the controller parameters.

Comparing the FP-GPC control law of equation (6) with the PID controller of equation (9), it is possible to obtain the corresponding calibration of the PID gains, hybridized with the FP-GPC, using the following expressions:

$$
\begin{aligned}
& K_{c}=\frac{-K_{\alpha} t_{0}\left(1-\alpha_{1}\right)\left(s_{1}+2 s_{2}\right)}{s_{0}+s_{1}+s_{2}}-r_{1} K_{c}(t-1)-\ldots-r_{n} K_{c}\left(t-n_{r}\right) \\
& T_{i}=-\frac{\left(s_{1}+2 s_{2}\right) T_{s}}{s_{0}+s_{1}+s_{2}} \quad, \quad T_{d}=-\frac{s_{2}}{s_{1}+2 s_{2}} T_{s}
\end{aligned}
$$

It is important to note from equation (10) that a time-varying proportional gain emerges to the PID controller tuning. This calibration rule of PID control gains is adjusted to optimize the closed-loop dynamic of the controlled process and can be implemented in a large number of digital devices and control loop applications. 


\subsection{Simulation Results to a Robot Arm Joint}

This essay is based on a practical simulation of a mechanical process called Robot Arm Joint (RAJ). The RAJ is an educational apparatus to emulate a manipulator arm controlled by a servo motor and has a potentiometer connected to the axis of the servo to measure the angular position (see Figure 4).

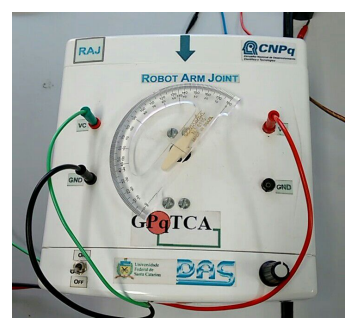

Figure 4: RAJ apparatus employed for control experiments.

The discrete model of the RAJ for a sampling period of $0.1 \mathrm{~s}$ is given in equation (11), it was obtained experimentally for a specific equilibrium point and the estimated transfer function is

$$
G_{p}\left(q^{-1}\right)=\frac{0.149 q^{-1}+0.094 q^{-2}}{1-1.008 q^{-1}+0.249 q^{-2}}
$$

Figure (5) shows the time response of the PID controller algorithm hybridized from the FP-GPC, for reference changes. The controllers have equivalent performance and good closed-loop dynamic. They are able to stabilize the system with small control variance, low output oscillation and minor measurement noise. Filter and FP-GPC parameters are tuned with $K_{\alpha}=0.85, \alpha_{1}=0.4, N_{y}=8, N_{u}=3, \lambda=8$ and sampling time of $0.1 \mathrm{~s}$. Based on equation (10), the PID gains are: $K_{c}=0.2726, T_{i}=0.1803$ and $T_{d}=0.0460$.
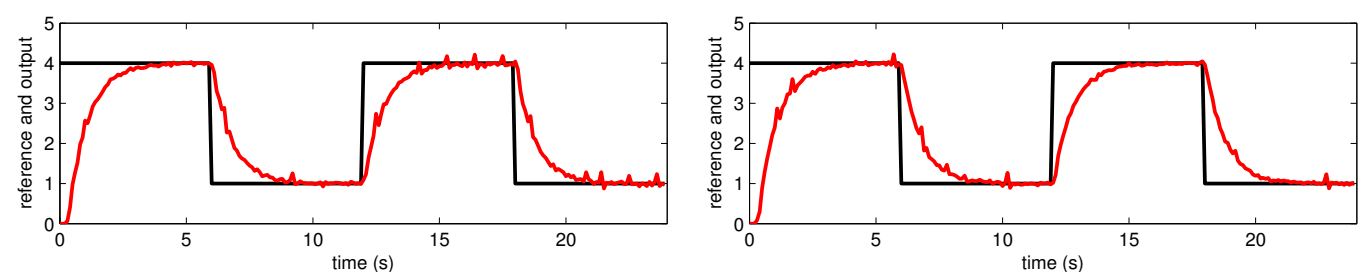

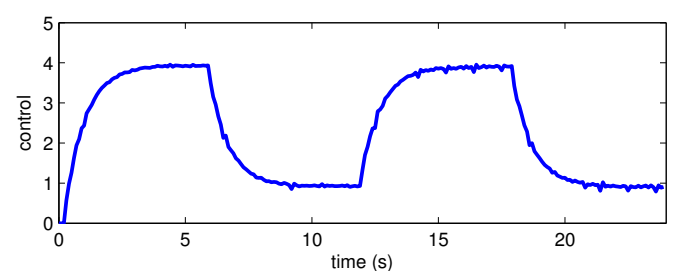

(a) FP-GPC

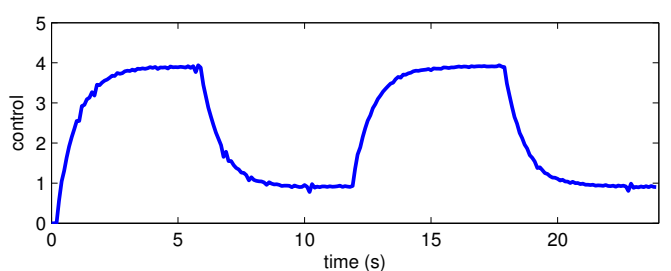

(b) PID-FP-GPC

Figure 5: System response to FP-GPC and PID controllers. 


\section{Conclusions}

We have proposed tuning sets for the IMC and PID controllers from the filtered positional GPC that has good closed-loop stability concerning setpoint tracking, disturbance rejection and model-plant mismatch.

Initially, the paper has derived the FP-GPC design with respect to the RST form. Next, the F-IMC has been hybridized to the FP-GPC. Numerical simulation was shown to an unstable and non-minimum phase plant to assess not only the FP-GPC design connected to the F-IMC implementation but also to demonstrate how the conventional IMC control structure can deal with unstable plants.

The last part of the paper has shown the standard PID controller, in the I+PD control structure, connected to the FP-GPC formalism and a PID tuning methodology was obtained. In order to assess the proposed tuning procedure in a practical application, a robot arm joint plant was employed. It was possible to observe that, in this case study, the obtained control results with the PID-FP-GPC tuning have shown a very satisfactory transient behavior.

\section{References}

[1] R. B. Araújo; D. C. Jeronymo and A. A. R. Coelho, Incremental and positional generalized predictive controller for offset free reference tracking, Brazilian Congress of Automatic Control, Brazil, (2014).

[2] D. W. Clarke; C. Mohtadi and P. S. Tuffs, Generalized predictive control - Part I: The basic algorithm, Automatica, pp. 137-148, (1987).

[3] M. S. Fadali and A. Visioli, Digital control engineering: Analysis and design, Elsevier, USA, (2009).

[4] D. Q. Mayne, Model predictive control: recent developments and future promise, Automatica, pp. 2967-2986, (2014).

[5] Z. Preitl; R. Bars; I. Vajk and R. Haber, Internal model representation for generalized predictive control with constraint handling, IEEE 4th International Conference on Intelligent Systems Design and Application, pp. 681-685, (2004).

[6] T. Sato and A. Inoue, Improvement of tracking performance in self-tuning PID controller based on generalized predictive control, International Journal of Innovative Computing, Information and Control, pp. 491-503, (2006).

[7] D. E. Seborg; D. A. Mellichamp; T. F. Edgar and F. J. Doyle, Process dynamics and control, John Wiley \& Sons, (2010).

[8] T. Yamamoto; T. Sato and A. Inoue, Improvement of tracking performance in designing a GPC-based PID controller using a time-varying proportional gain, Transactions on Electrical and Electronic Engineering, pp. 438-441, (2006). 